\title{
INTEGRATED ANALYSIS OF ENVIRONMENT-DRIVEN OPERATIONAL EFFECTS IN SENSOR NETWORKS ${ }^{\dagger}$
}

\author{
Alfred Park, Kalyan Perumalla* \\ Computational Sciences and Engineering \\ Oak Ridge National Laboratory \\ Oak Ridge, Tennessee, USA \\ *Corresponding Author: perumallaks@ornl.gov
}

\section{KEYWORDS}

Sensor Networks, Simulation, Plume Models

\begin{abstract}
There is a rapidly growing need to evaluate sensor network functionality and performance in the context of the larger environment of infrastructure and applications in which the sensor network is organically embedded. This need, which is motivated by complex applications related to national security operations, leads to a paradigm fundamentally different from that of traditional data networks. In the sensor networks of interest to us, the network dynamics depend strongly on sensor activity, which in turn is triggered by events in the environment. Because the behavior of sensor networks is sensitive to these driving phenomena, the integrity of the sensed observations, measurements and resource usage by the network can widely vary. It is therefore imperative to accurately capture the environmental phenomena, and drive the simulation of the sensor network operation by accounting fully for the environment effects. In this paper, we illustrate the strong, intimate coupling between the sensor network operation and the driving phenomena in their applications with an example sensor network designed to detect and track gaseous plumes.
\end{abstract}

\section{INTRODUCTION}

Sensor networks are being designed for use in a variety of applications. Some of the applications of interest to us belong in the areas of border surveillance, chemical/biological agent monitoring, homeland security concerns in critical infrastructures and public/government assets. Design and development in these and other areas have been advancing the scalability and effectiveness of the sensor networks. As the complexity and scale of sensors networks increase, so does the need for their effective design and analysis. The complexity of overall sensor network operation is increased with the growing sophistication of data fusion techniques, tracking algorithms, sensor device capabilities, network device characteristics and amount of relevant detail in the phenomena being tracked.
The competing concerns of improved fidelity and performance abound in sensor network operations. While frequent sensor readings can help deliver better accuracy, they can strain the network and also compete with other sensors for network bandwidth. Increasing the wireless radio range of sensor nodes can help decrease the number of hops to a basestation and reduce latency, but it introduces greater interference and/or collisions in radio transmissions among neighbors. Adhoc packet routing, via a dynamically established mesh, can help alleviate set up efforts, but can also introduce complex delay and loss patterns into the sensor reading transmissions. Data fusion algorithms [1] can help alleviate bandwidth requirements, but they are subject to various types of assumptions including those about both the sensed phenomena and the inter-sensor connectivity arrangements.

Critical sensor networks deployments often need an accurate a priori understanding of the expected performance of the deployment. Depending on the type of the network, an estimate of the "average case" may be sufficient. In other contexts, it may be necessary to plan for the unanticipated "worst case." It becomes critical to take into account the spectrum of possible scenarios under which the sensor network deployment will have to operate. Limiting the testing to only a few scenarios could expose the application to unexpected operation, sometimes incurring a large amount of risk and very severe consequences. Our central contribution is demonstrating the specific need for higher-fidelity joint simulations of both the sensor network and the wider-area driving scenarios. We show in our simulation results that this exposes behaviors that traditional approaches (e.g., those that assume point processes for arrival rates, or those that assume nonglobal effects) may not capture.

\section{Example: Sensor Networks For Plume Detection}

Here, we focus on a type of sensor network that exhibits a critical type of operation and hence requires careful analysis of the range of relevant scenarios. Specifically, we consider sensor networks that are designed to sense air-borne chemical/biological agents and rapidly send the sensed observations to basestations/control centers

\footnotetext{
† This manuscript has been authored by employees of UT-Battelle, LLC, under contract DE-AC05-00OR22725 with the U.S. Department of Energy. Accordingly, the United States Government retains and the publisher, by accepting the article for publication, acknowledges that the United States Government retains a non-exclusive, paid-up, irrevocable, world-wide license to publish or reproduce the published form of this manuscript, or allow others to do so, for United States Government purposes.
} 
[2]. Clearly, reliable operation of such networks can be critical, especially if deployed for detecting lethal agents.

Although there has been much effort to track entities using sensor networks [3-5], another important application is the use of such networks for fast and accurate inverse tracking of the location of bombs upon explosion [6]. Speed and accuracy of detection/tracking of the sources is obviously of critical interest. Unfortunately, the problem is characterized with numerous dimensions and includes a composition of many non-linear processes. Simulation-based evaluation of the design is often the only method for analyzing and estimating the overall performance of the network. Moreover, due to the tight dependencies among the elements, it is necessary to capture their intertwined operation within the same simulation, rather than as a composition of results from simulation of independent elements. For example, the intensity and frequency of wireless radio communication by sensor nodes is directly dependent on the phenomenon being observed, and activated by threshold-crossing sensor readings. Consequently, separating the simulation of the phenomenon from the simulation of the sensor network devices is not warranted and may yield misleading results.

\section{EVALUATION PLATFORM}

In order to satisfactorily evaluate the range of scenarios to which the plume detection sensor networks would be subjected, we adopt a high-fidelity simulation approach. A high resolution plume generation and dispersion model is used to simulate the phenomenon being sensed and tracked. The sensors are modeled in full detail at the level of analog-to-digital (A/D) conversion. The sensor network nodes connected to the sensors are simulated at the highest resolution possible, namely, by emulation of actual operating system and program execution. Wireless communication is simulated at bit level, and wireless signals are simulated using detailed interference, collision and loss models. Network operation is simulated with actual ad-hoc routing protocols executing on all sensor nodes.

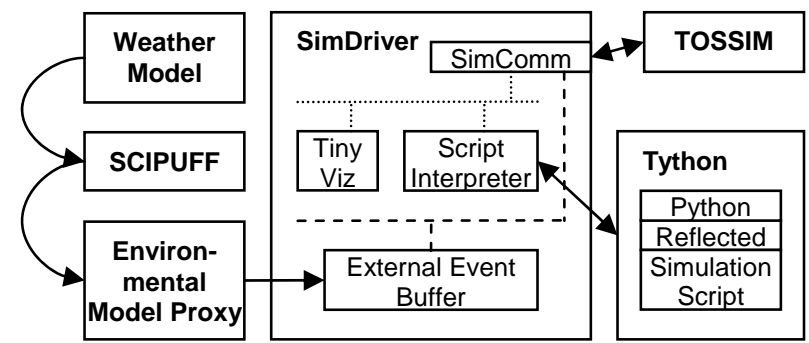

Figure 1: Experimental Infrastructure

The experimental platform illustrated in Figure 1 is composed of two high-fidelity simulators that a central SimDriver module brings together: (a) a detailed plume generation and dispersion simulator, and (b) a detailed sensor network simulator. The output generated by the plume dispersion model is fed into the sensor network simulator to drive the sensors and sensor network operation. The high fidelity atmospheric dispersion simulation model generates concentration levels at sensor locations. These values are used to feed the sensor A/D interfaces at the sensor nodes positioned at the corresponding locations.

\section{Second-order Closure Integrated Puff (SCIPUFF)}

The high-fidelity plume dispersion simulator that we employ is the Second-order Closure Integrated Puff modeling tool $[7,8]$. SCIPUFF is a Lagrangian puff dispersion model where "the turbulent diffusion parameterization is based on second-order turbulence closure theory, which relates the dispersion rate to velocity fluctuation statistics.” SCIPUFF is capable of modeling three-dimensional, time-dependent concentration fields, in terms of "puffs" that are split and/or merged over time as necessary. Details such as wind shear effects are taken into account. These and several other modeled mechanisms allow the SCIPUFF to describe complex flow effects on dispersion, including circulation affected by terrain.

The SCIPUFF model has been widely used across many different domains and organizations. The U.S. Defense Threat Reduction Agency (DTRA) has incorporated the SCIPUFF model into the U.S. government-controlled Hazard Prediction and Assessment Capability (HPAC) software. Aside from its use by the U.S. Army for military evaluations, SCIPUFF has also been recommended by the U.S. Environmental Protection Agency (EPA) as an alternative model for some regulatory applications on a case-by-case basis.

\section{TOSSIM and Tython}

The TinyOS Simulator (TOSSIM) is a sensor network simulator based on the TinyOS operating system for wireless embedded sensor devices called motes [9, 10]. Motes typically have constrained communication bandwidth, limited computational power, and finite energy resources, but have relatively low power requirements. TOSSIM is unique compared to other sensor network simulators such as GTSNetS [11], J-Sim [12], SENSE [13], SensorSim [14], and TOSSF [15] in that the simulation code is built directly from the same code that can be deployed to real motes. This allows application developers the expanded capability of testing unmodified application implementations in simulation. Similar frameworks include EmStar [16] and EmTOS [17]. TOSSIM includes a high fidelity bitlevel simulation of the radio stack which allows modeling of collisions at the bit-level and accurately capturing the hidden-terminal phenomena.

Tython [18] provides control of TOSSIM simulations via a scripting interface. Tython is built on Jython, a Java implementation of the Python language, allowing users to exploit the full expressive power of Python to access the entire TinyOS Java tool chain. This allows powerful runtime manipulation of TOSSIM simulations such as dynamically adding external 
stimuli. We use this interface to incorporate SCIPUFF readings as input to simulated sensors attached to TOSSIM motes.

For the following experiments, we gathered concentration values and time information from SCIPUFF and directed it to a proxy in Tython, as shown in Figure 1. We inject this data with interrupts into TOSSIM buffered accordingly in Tython for processing as future discrete events.

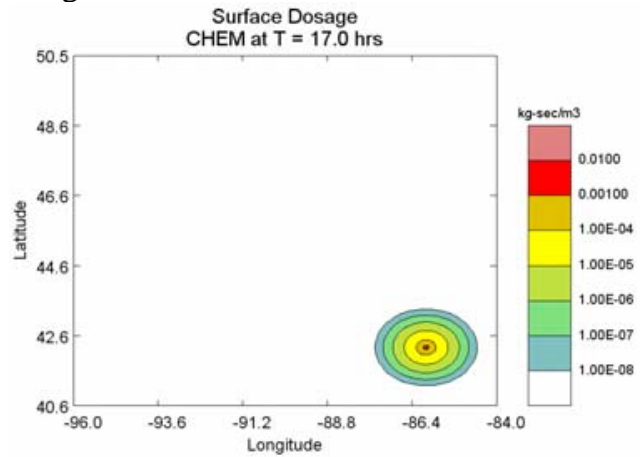

Figure 2: Plume Propagation with No Winds

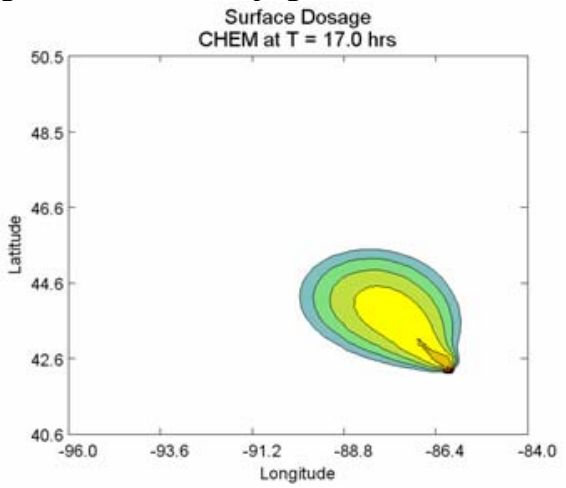

Figure 3: Plume Propagation with (Moderate) Winds

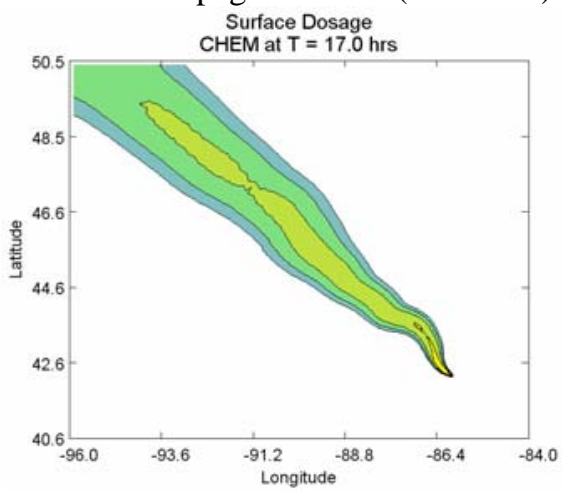

Figure 4: Plume Propagation with Turbulent Weather

\section{Sensor Application and Network Routing}

The TinyOS NesC application used in the study is one that activates a generic sensor and samples these values from the A/D Converter (ADC) periodically. Each sensor employs data fusion by sending average sample values over time to the base station node. We configured an ad-hoc multi-hop routing implementation using shortest-path-first and activate two-way link estimation to set up ad-hoc routes and route packets from source nodes to the base station node [19]. Due to limited wireless bandwidth, sensors sample from the
ADC every 250 milliseconds and store the sampled value into a circular buffer of length 8 . Once the circular buffer is filled, a packet is emitted containing the average value of all readings in the buffer. This translates into one packet sent every two seconds, as to not exceed the bandwidth available for both the application and the multi-hop ad-hoc routing protocol.

\section{EXPERIMENTAL STUDY}

The plume detection and tracking application is a fairly complex one, with many dimensions and parameters. Here we focus our study on a subset of the dimensions, to highlight the amount of variability of sensor network performance with varying set of scenarios and the need for careful and methodical evaluation of scenarios in a real-life deployment.

\section{Scenario Parameters}

\section{Weather and Dispersion Effects}

In our experiments, the weather model in SCIPUFF was varied with three representative scenarios: no winds, some winds, and turbulent weather conditions, whose snapshots are illustrated in Figure 2, Figure 3 and Figure 4 respectively. The weather model scenarios respectively exhibit no precipitation and no winds, no precipitation with variable light to moderate winds up to $8 \mathrm{mph}$, and heavy rain with variable winds up to 40 mph respectively. Temperatures for all cases fell between 70 to $77{ }^{\circ} \mathrm{F}$. The no winds case is used to represent a base model as shown in Figure 2 due to near uniform spread of the gas in all directions indicating a simple dispersion model which is sufficient for comparing to more complex scenarios.

The SCIPUFF model generates concentration values for a single source release with a duration of 300 seconds in the middle of the area modeled, with a release time at 300 seconds. The initial 300 seconds allow the network routing protocol to build routes to the base station.

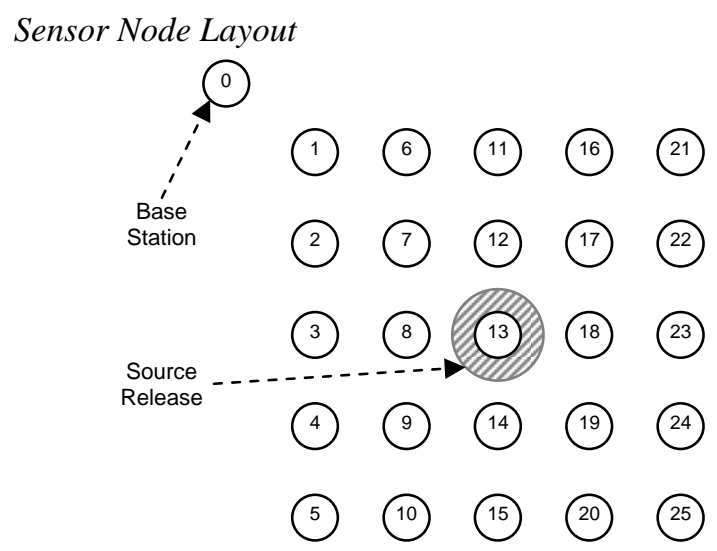

Figure 5: A 5×5 Sensor Network Layout Two flat geographic areas were modeled: a dense area measuring $131.23 \mathrm{ft}^{2}$ and a sparse area measuring $196.85 \mathrm{ft}^{2}$. For each of these areas, the sensors were arranged as a $5 \times 5$ grid of sensor motes at ground level 
as shown in Figure 5. Motes are numbered contiguously, with zero as the base station, one on lefttop corner, 5 on left-bottom corner, 21 in right-top corner and 25 at right-bottom corner (mote 13 is at the source).

\section{Wireless Communication Effects}

The radio propagation model was varied for each case using either a binary disc model or an empirical lossy model. In the binary disc model, any pair of nodes within 50 feet of each other would be guaranteed to receive packets from each other (assuming no collisions during the packet transmission). In the empirical lossy model, two-way loss rates are assigned from observed values as assigned by TOSSIM's LossyBuilder program.

\section{Simulation Results}

\section{Sensor Reading Variation due to Weather}

In the sparsely populated sensor node grid configuration (sparse $5 \times 5$ ), a maximum of one other node can hear packet transmissions from the sender in each direction. This configuration allows analysis of weather effects as perceived by the base station with minimal impacts from the wireless network due to packet loss from collisions.

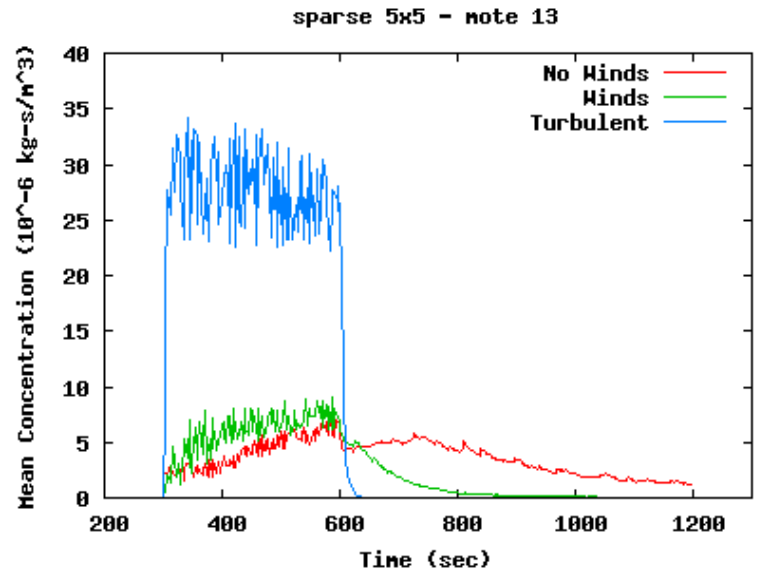

Figure 6: Sensor Readings at Release Source

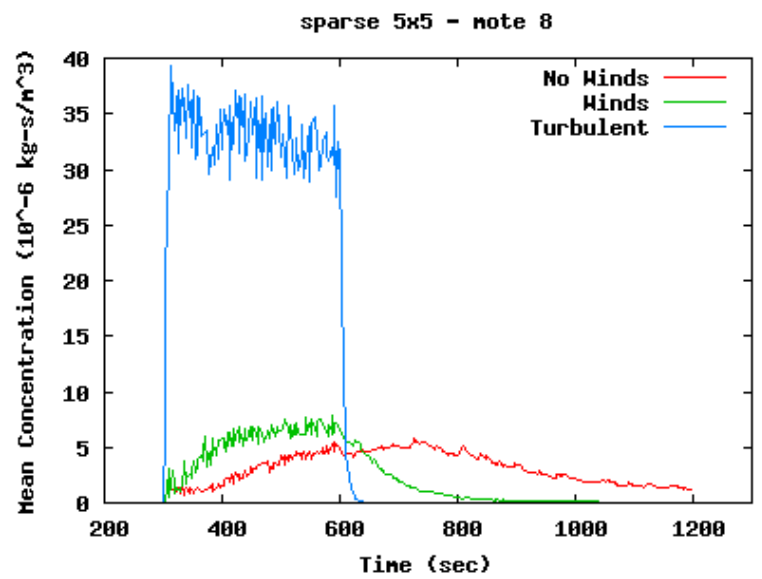

Figure 7: Sensor Readings 32.81ft from Source in the General Wind Direction
One of the clear observations from the experiments is the significant effect of weather on the phenomenon sensed at the base station. The weather is varied, as mentioned before, into three categories roughly representing calm, moderate and turbulent weather. Figure 6 shows mean concentration readings for the gas of interest for each of the three weather conditions, corresponding to the values sent by the mote closest to the plume source. The sensor readings as received by the base station are plotted over time.

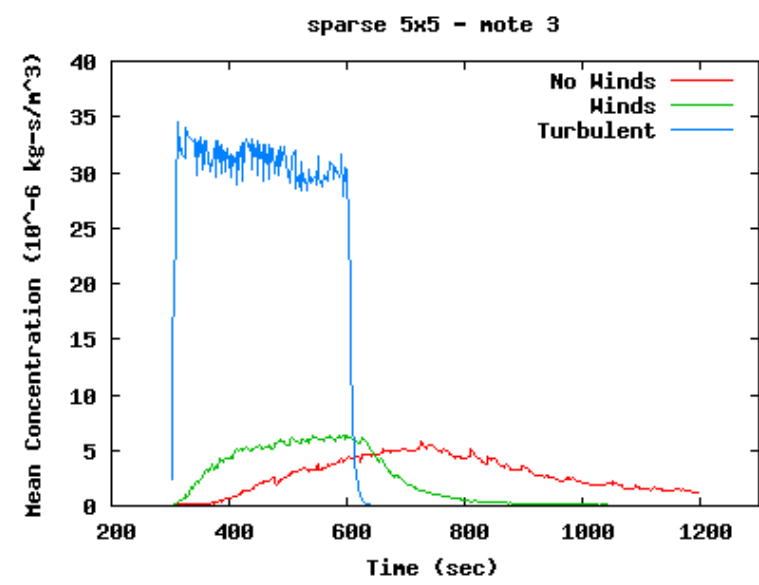

Figure 8: Sensor Readings 65.61ft from Source in the General Wind Direction

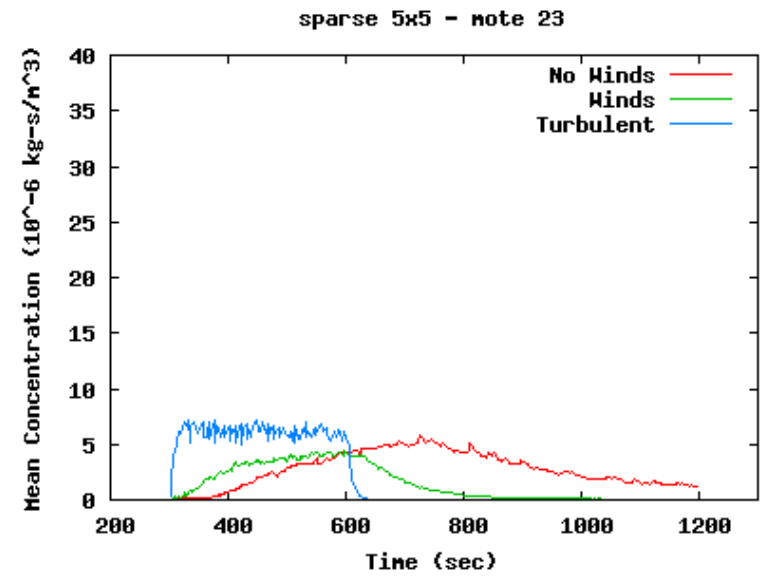

Figure 9: Sensor Readings 65.61ft from Source in Opposite Wind Direction

As expected, the no winds case shows a gradual rise in observed readings due to plume release and then a steady decline due to plume dispersion. In the winds case, the concentration of the gas rises faster with greater variability in the readings. However, after the gas release stops at 600 seconds, the readings decline towards zero faster than the no winds case due to dispersion of the gas aided by winds. The mean concentration rises sharply with high fluctuation for the duration of the release in the turbulent case due to high winds and heavy downpour dispersing the gas quickly. Upon end of the release, mean concentration values for the gas decline rapidly, again due to the high winds, reaching undetectable levels far quicker than the no winds or the winds cases. 
Figure 7 and Figure 8 show concentration values directly to the west of the sensor at the release source and further west, respectively. The wind in this scenario is blowing west for both the winds and the turbulent cases. These figures show the spread of the gas to these sensors in a similar pattern to the sensor at the release source, however slightly time-delayed. Figure 7 shows higher concentration values in the turbulent case due to the high winds dispersing the gas of interest from the source to mote 8 .

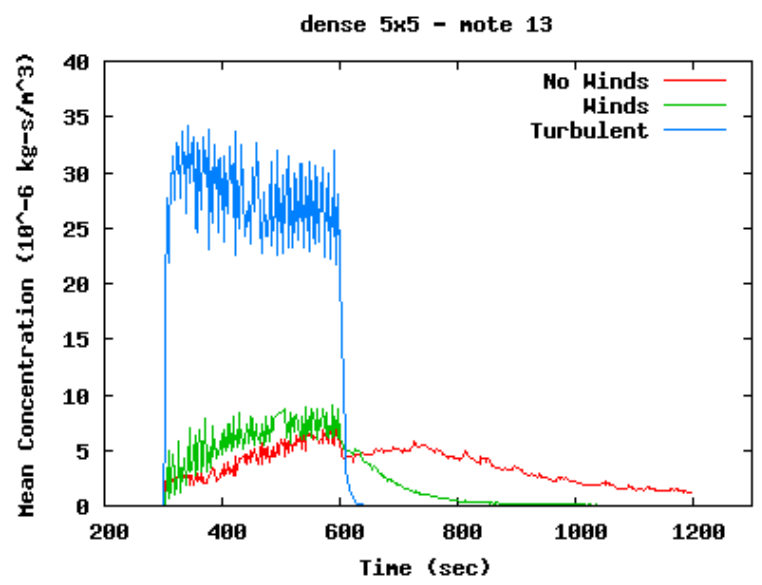

Figure 10: Sensor Readings at Release Source

Figure 9 shows sensor readings for the mote situated furthest east of the source, opposite of the general wind direction. Mean concentration values are lower for the winds case and significantly lower for the turbulent case than observed at the source release and at mote locations which fall in the general wind direction.

From these observations, it is clear that weather has a pronounced effect, and hence, accurate evaluation of the sensor network requires one to evaluate the network under a range of weather conditions via detailed simulation.

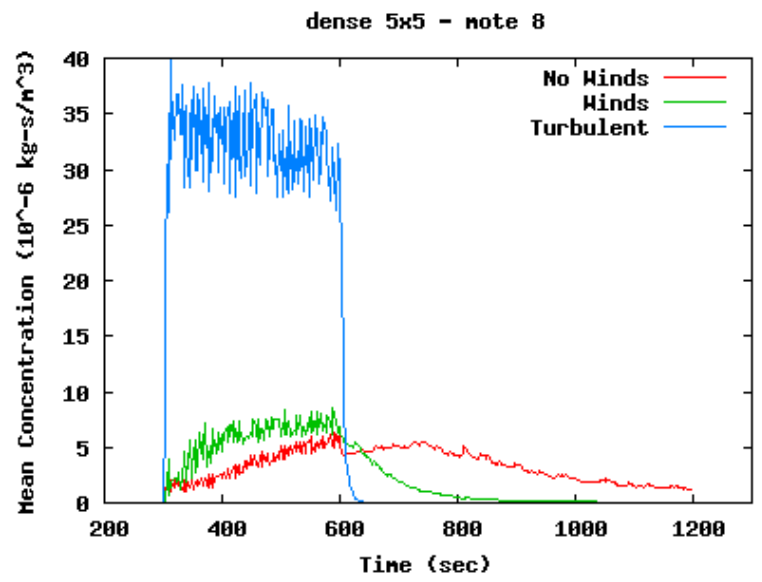

Figure 11: Sensor Readings 21.87ft from Source in the General Wind Direction

Network Performance Variation due to Node Density

In the densely packed sensor grid (dense $5 \times 5$ ), sensor nodes can be in range of up to two other sensor nodes in its broadcast radius. Although this can reduce the number of hops a packet must traverse to the base station node, the probability of bit collisions increases.
This scenario provides some insight into network effects on readings received by the base station as compared to the sparsely connected sensor network.

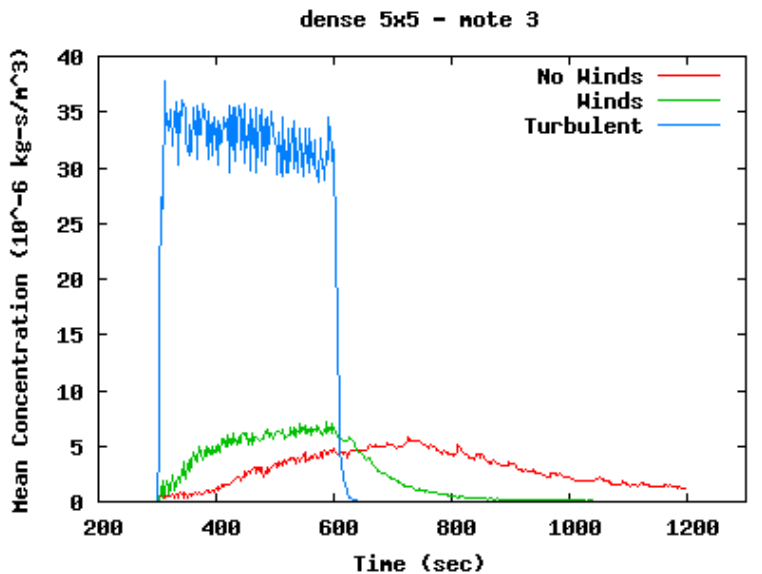

Figure 12: Sensor Readings 43.74ft from Source in the General Wind Direction

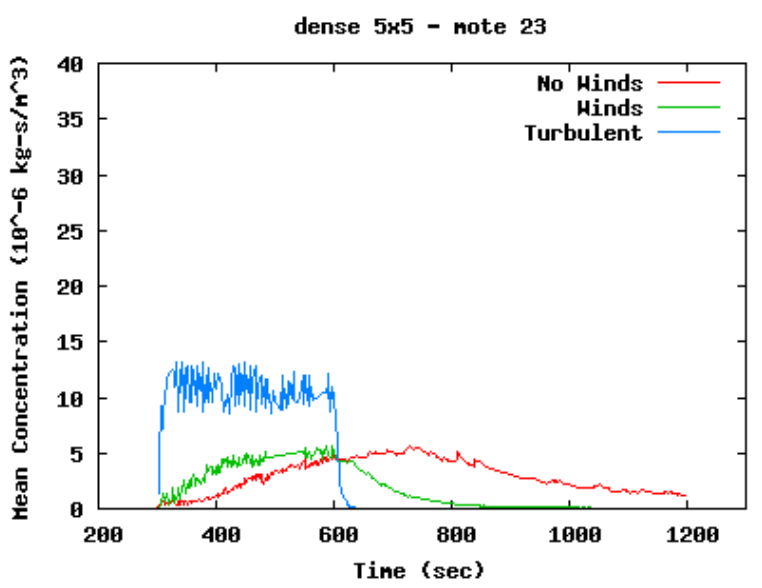

Figure 13: Sensor Readings 43.74ft from Source in Opposite Wind Direction

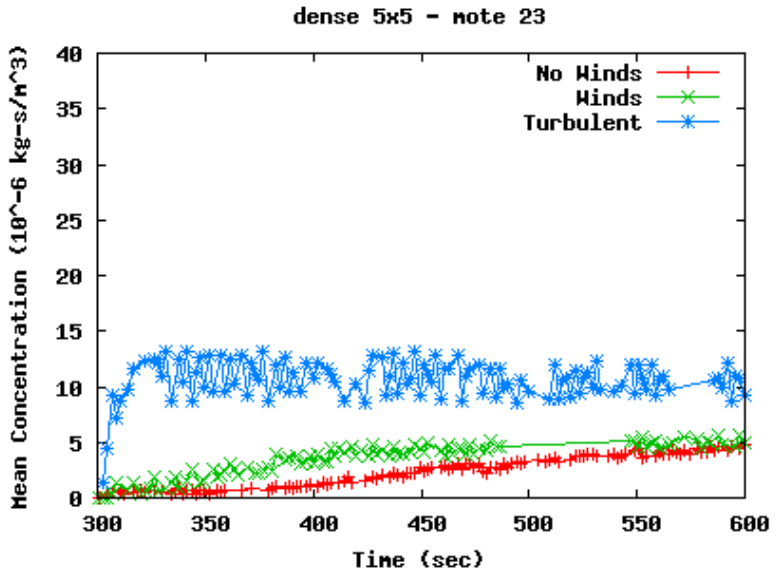

Figure 14: Sensor Readings showing the Effect of Packet Loss

The sensor readings of the dense network show similar patterns to the sparse sensor network grid configuration although with higher concentration values due to reduced inter-sensor distance to the source than the sparse case as shown in Figure 10, Figure 11, Figure 12, and Figure 13. In each of these cases, we can observe small anomalies in the readings received by 
the base station. There are small to large gaps of in sensor readings due to packet losses from bit collisions that were not present in the previous sparse $5 \times 5$ scenarios. However, observations indicate small anomalies in the readings received by the base station.

Figure 14 shows the source release time in more detail, illustrating large periods of time where no data for mote 23 was received by the base station. In the winds case, no sensor readings were received for approximately 60 seconds, a significant portion of the release time. If any fluctuations in sensor reading had occurred during this time frame, they would not have been perceived by the base station. These gaps in sensor readings are due to packet losses from bit collisions that were not present in the sparse scenarios.

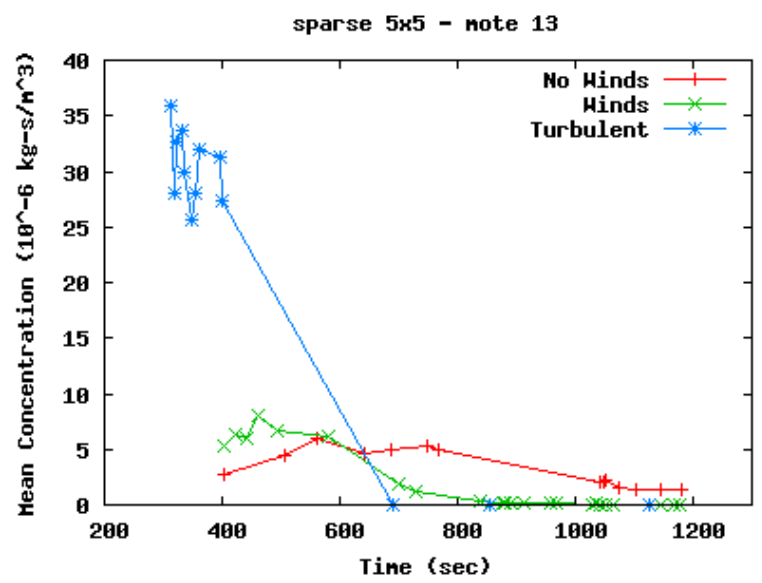

Figure 15: Sensor Readings at Release Source

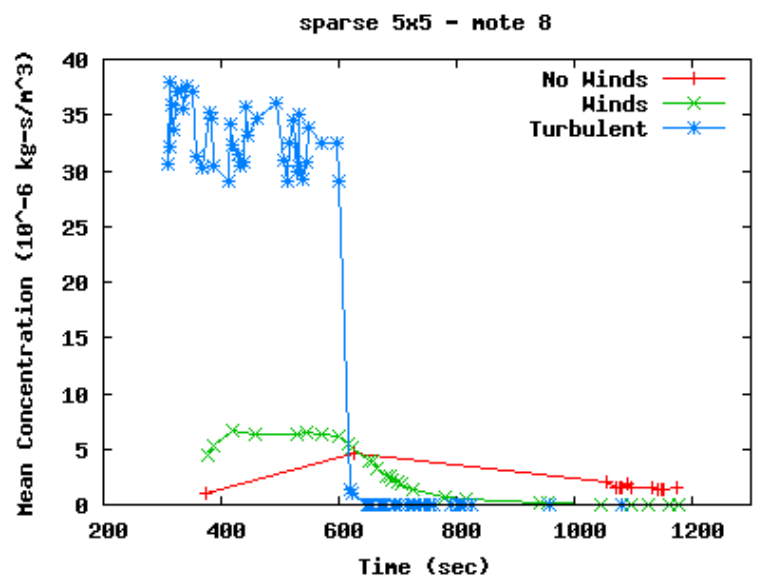

Figure 16: Sensor Readings 32.81ft from Source in the General Wind Direction

\section{Wireless Communication and Network Effects}

Binary Disc Radio Propagation Model: Under the bestcase network operation where packets can be received by other nodes if within 50 feet assuming no bitcollisions occur during packet transmission, weather effects on sensor readings can be analyzed with minimal impact from network effects by using the binary disc model as illustrated in the previous scenarios.

Empirical Lossy Radio Propagation Model: The following scenarios evaluate the impact of a lossy radio model exhibiting bit-error rates with some probability mirroring real-world observations. This is achieved by sampling by distance from a Gaussian packet loss probability distribution matching observed data [10].

Figure 15, Figure 16, Figure 17, and Figure 18 show dramatically different results than what was observed in the binary disc model. Although the rough outline of trends can be determined from the figures, many critical points are missing from each sensor node location. For instance, at the source release mote 13, there is no indication of a rise in concentration, or a rapid decrease in concentration for the turbulent case as was observed in Figure 6. In the no winds and winds cases for mote 13, no data is received until 100 seconds after the source release begins.

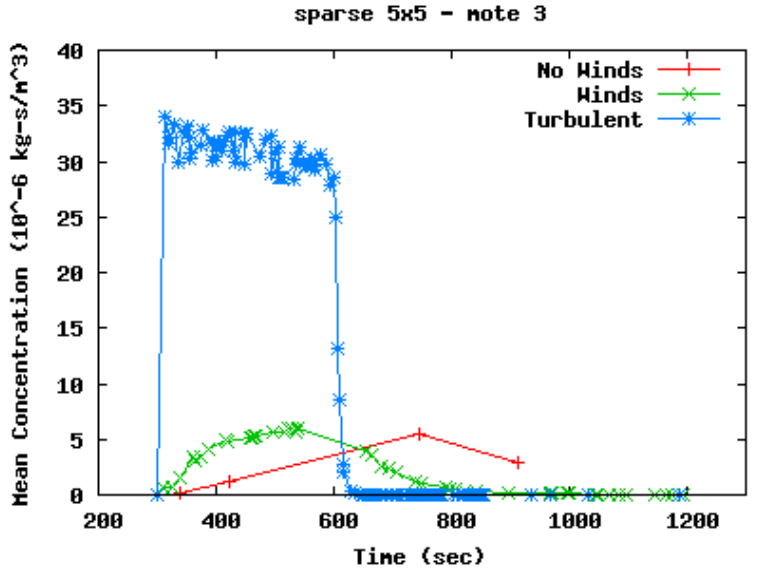

Figure 17: Sensor Readings 65.61ft from Source in the General Wind Direction

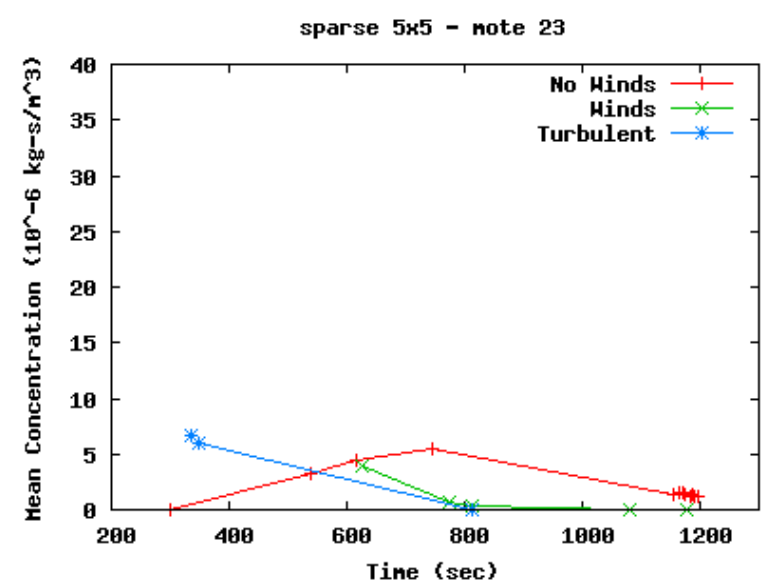

Figure 18: Sensor Readings 65.61ft from Source in Opposite Wind Direction

Figure 18 shows no discernable trends for the winds and turbulent cases as the data received by the base station is too sparse except for the no winds case. The low number of received packets is due to the distance that mote 23 is away from the base station node requiring a minimum of five hops in the sparse grid. Each hop incurs a probability of transmission failure either due to collisions or corruption at the bit-level. The relationship between distance and number of packets received is observed in Figure 15, Figure 16, Figure 17, and Figure 18 with the base station receiving more packets from closer nodes such as motes 3 and 8 than nodes further away such as 13 and 23. 


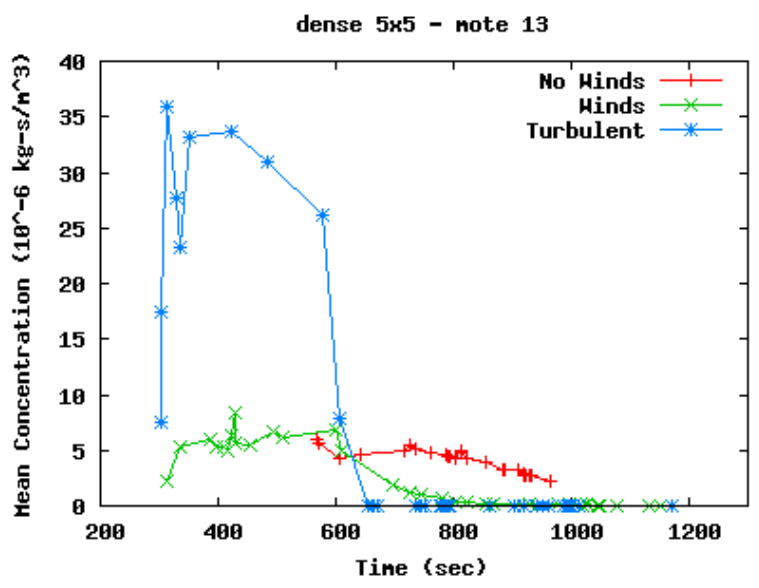

Figure 19: Sensor Readings at Release Source

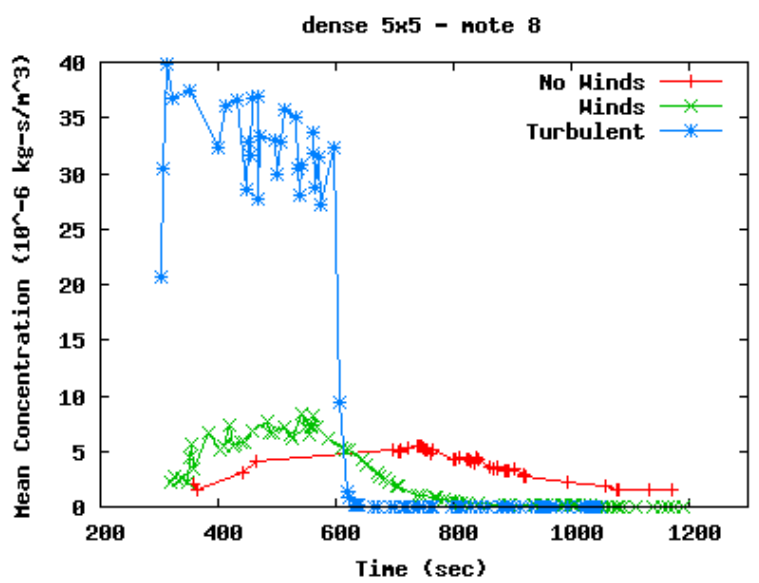

Figure 20: Sensor Readings 21.87ft from Source in the General Wind Direction

dense $5 \times 5$ - note 3

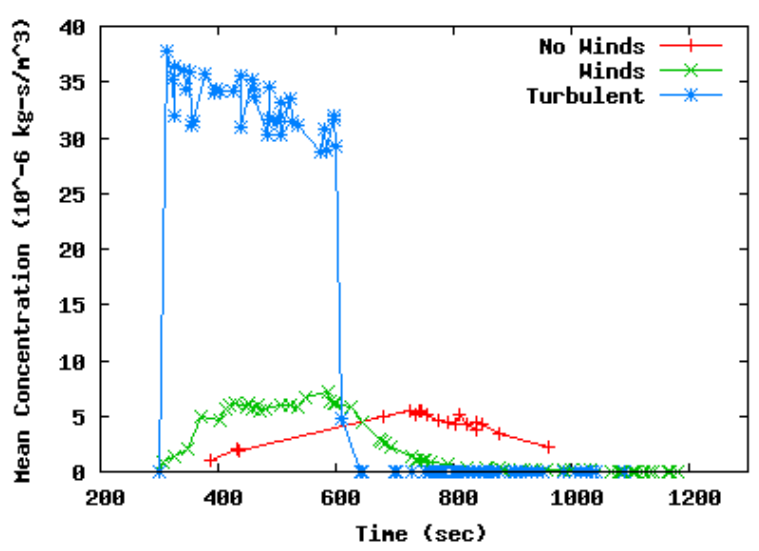

Figure 21: Sensor Readings 43.74ft from Source in the General Wind Direction

With the decrease in distances between motes in the dense scenarios, the bit-error rate decreases resulting in higher reception rates across all motes as illustrated in Figure 19, Figure 20, Figure 21, and Figure 22. Although this improves the rate at which packets are received by the base station from all sensor nodes, there are deficiencies still present such as in the no winds case in Figure 19 where the first data point is received near the end release time and thus no noticeable pattern of concentration increase is observed and in Figure 22 where no noticeable pattern of concentration increase then decrease is observed in the no winds case.

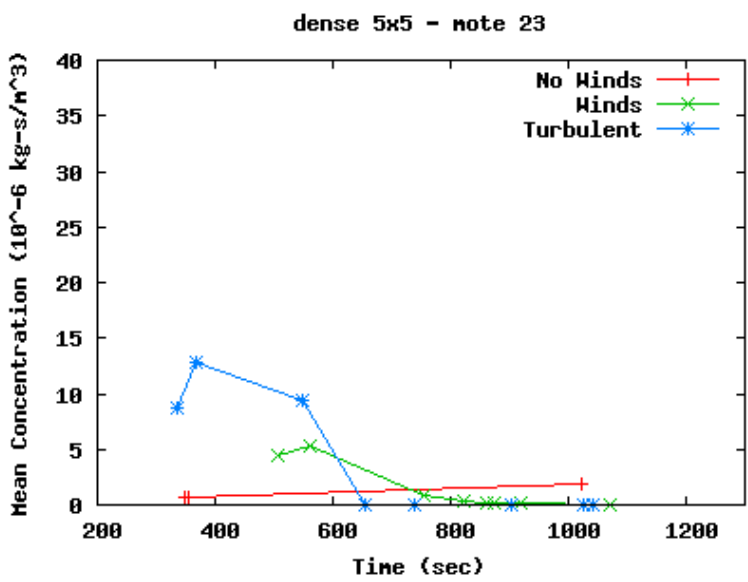

Figure 22: Sensor Readings 43.74ft from Source in Opposite Wind Direction

A high fidelity sensor network simulator with realistic radio propagation models can reveal phenomena such as low packet reception as seen in the empirical lossy cases. These effects combined with different weather models from the environmental simulator produces highly variable results as perceived by the base station.

Effects of Sensor Network Application Implementation

We have observed the effect of weather, inter-sensor distance, and radio propagation models have had on perceived concentration activity. It is also of interest to examine the side-effects of how the data is modeled by the sensor network simulator due to the design of the sensor application.

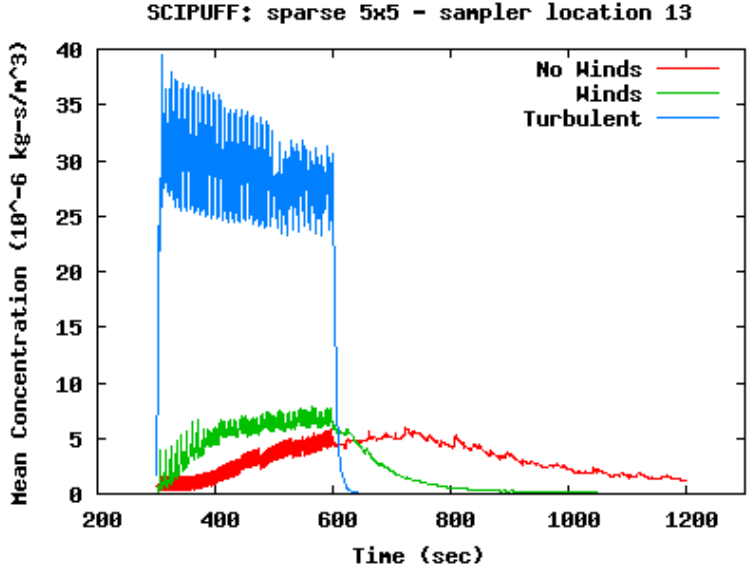

Figure 23: SCIPUFF Readings at Release Source for Sparse $5 \times 5$ Grid

Figure 23 shows the corresponding concentration levels for mote 13 in the sparse $5 \times 5$ scenarios (Figure 6 ) as reported by SCIPUFF and unfiltered by the sensor network simulator. The general mean concentration rise and subsequent drop off is represented correctly in the sensor readings reported by mote 13 to the base station. The initial concentration spike seen in Figure 23 for the turbulent case is not seen in Figure 6 due to the data fusion employed on the sensor nodes. Actual sensor values are not reported, instead every 8 concentration 
readings are averaged, and this value is sent back to the base station.

SCIPUFF: dense $5 \times 5$ - sanpler location 13

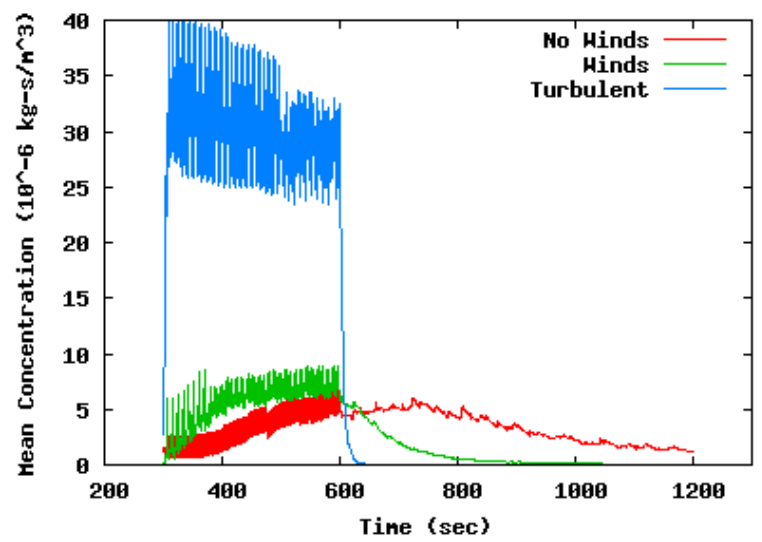

Figure 24: SCIPUFF Readings at Release Source for Dense $5 \times 5$ Grid

Figure 24 displays readings from the environmental simulator for the dense $5 \times 5$ scenarios. It is interesting to see much less variability, and hence, lower maximum values in the concentration values than when the data is filtered through the sensor network simulator as seen in Figure 10. By utilizing a high-fidelity realistic sensor network simulator which allows application implementation simulation (e.g., TOSSIM), we can examine the effects such as data fusion techniques on sensor information received by the base station.

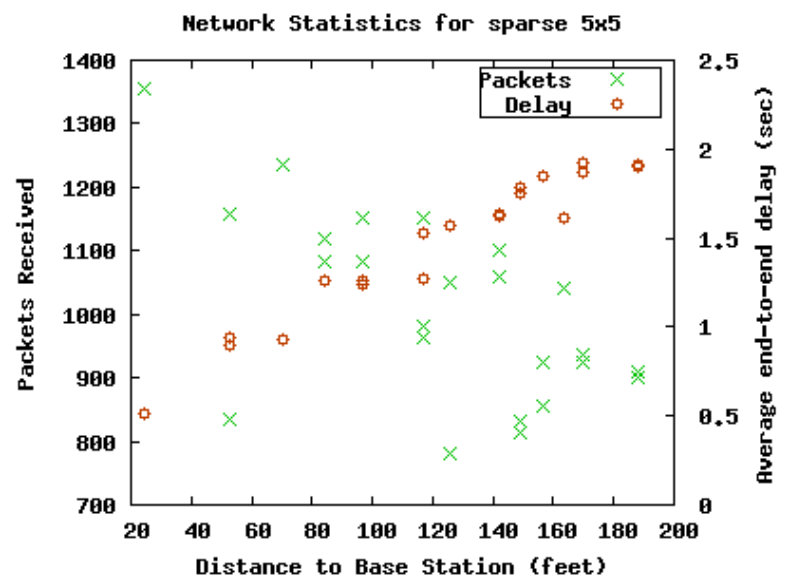

Figure 25: Binary Disc Model for Sparse 5×5 Grid

\section{Aggregate Network Performance}

Although some of the network effects were observed when visualizing concentration values for various cases, analysis of the interplay and effects of network layout, sensor node promixity, and constrained bandwidth is of interest to evaluate the cumulative impact of the simulation results.

For the binary disc radio propagation models, Figure 25 and Figure 26 show an expected inverse relationship in packets received as the absolute distance between the base station and the sensor location increases. Moreover, the observed average end-to-end delay increases as distance increases between the base station and mote. Figure 27 and Figure 28 illustrate a rapid decline in packet reception rates as the distance between sensor location and base station increases for the empirical lossy radio propagation models. This is due to the added bit-error rates which can cause packets to be corrupted and dropped in addition to normal packet loss due to collisions.

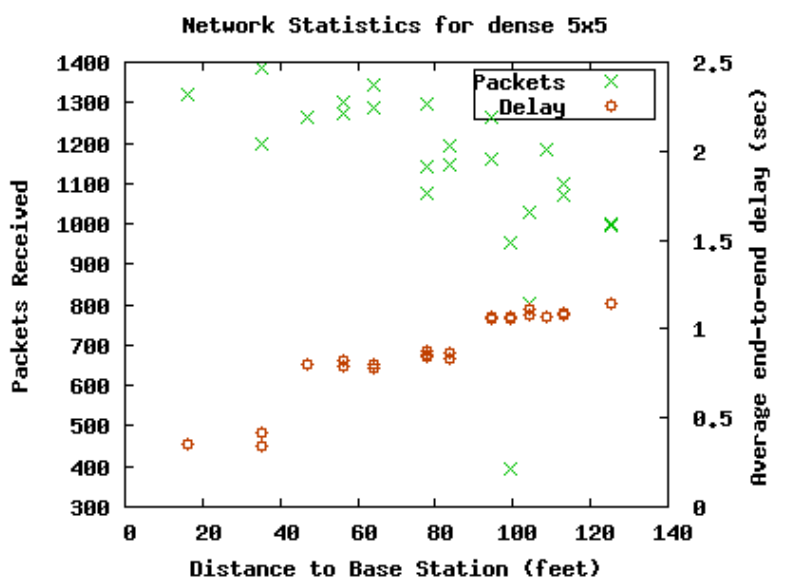

Figure 26: Binary Disc Model for Dense 5×5 Grid

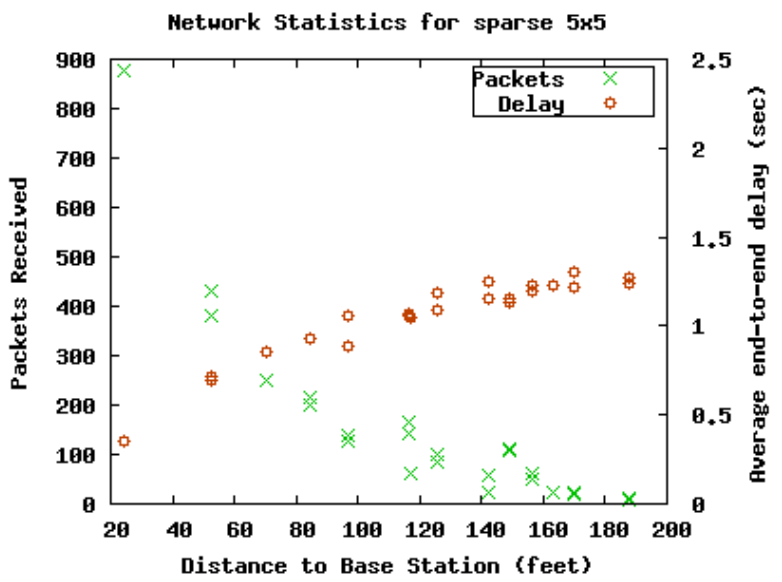

Figure 27: Empirical Lossy Model for Sparse 5×5 Grid

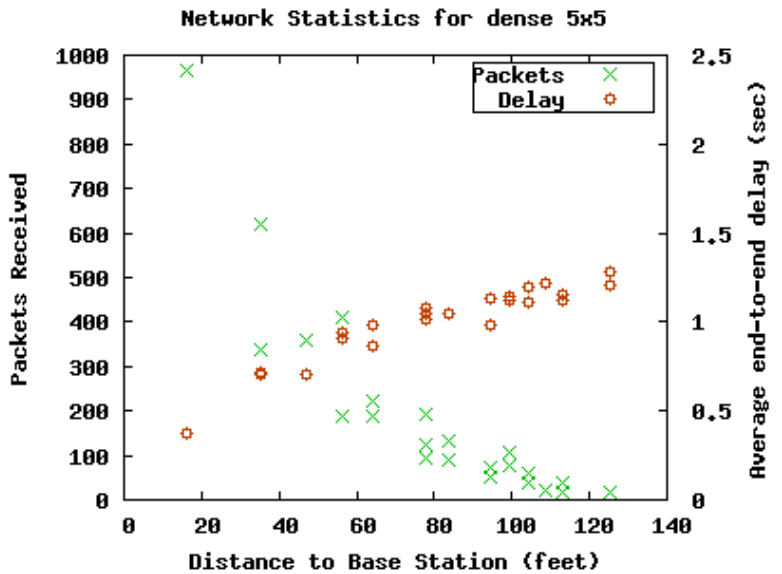

Figure 28: Empirical Lossy Model for Dense $5 \times 5$ Grid

An interesting phenomenon that only occurs in the sparse $5 \times 5$ binary disc radio propagation model is the set of observations with 1.5 second average end-to-end delays (Figure 25). This pattern is observed due to $100 \%$ accurate packet reception for nodes at the edge of radio range assuming no collisions, while in the empirical lossy models, many of these packets would be lost due to bit errors as shown in Figure 27. Using 
models that compromise accuracy, such as the binary disc model, can display anomalies such as receiving packets which would, in reality, have very small probability of successful reception.

From these observations, sensor network application implementations could incorporate a sophisticated packet acknowledgement and retransmission scheme to improve network performance and ultimately more accurate sensor readings.

\section{ATMOSPHERIC TRANSPORT AND DISPERSION MODELS}

Currently, there are many different atmospheric transport and dispersion models being developed and used for plume propagation modeling. The following is a brief overview of three of the many categories of such models being employed today.

\section{Gaussian Plume Models}

The Gaussian plume model [20] is a widely used computational approach to calculating concentration levels of a compound of interest (e.g., gas, aerosol, etc.). This model assumes that the horizontal and vertical dispersion is modeled as a normal Gaussian distribution with the center of the plume being the maximum concentration. Constant meterological conditions are also assumed producing straight-line trajectories, however, recent improvements in Gaussian plume models have the ability to incorporate mixing, convection, and terrain effects. Examples of atmospheric transport and dispersion models utilizing Gaussian plume techniques include AERMOD [21], CAMEO/ALOHA [22], and ISC3 [23].

\section{Lagrangian Puff Models}

Instantaneous sources are modeled as puffs for atmospheric transport and dispersion models leveraging Lagrangian techniques. These models are not exclusively restricted to instanteous sources as a sequence of puffs can be modeled achieving the same release over time as a continuous source. Puffs can expand at their center due to wind effects and stability. Additionally, puffs can be split or merged as necessary over time. Lagrangian puff models are usually best suited for region-scale areas with timescales of hours to days. Examples of Lagrangian puff models include SCIPUFF/HPAC [7, 8], HYSPLIT [24], CALPUFF [25], and HOTMAC/RAPTAD [26].

\section{Computational Fluid Dynamics and Large Eddy Simulations}

Atmospheric transport and dispersion models utilizing Large Eddy Simulation (LES) [27] and Computational Fluid Dynamics (CFD) techniques are especially useful for environmental simulations with small temporal and spatial scales. These models perform exceptionally well for relatively smaller areas (than areas modeled by Lagrangian plume models) with buildings, street canyons, and noisy dispersion (e.g., dispersion affected by traffic and HVAC systems) as found in urban environments. Examples of models using these approaches include FEM3C [28] and FEFLO-URBAN [29].

One can envision the ability to interchange different atmospheric transport and dispersion models in the experimental framework (Figure 1) to achieve varying levels of fidelity and accuracy depending upon the temporal and spatial resolution as required by the scenario. Different atmospheric models may be more suitable for a particular scenario such as a simulation model specifically tailored for small-scale urban environments with tall buildings and a complex road network.

\section{SUMMARY AND FUTURE WORK}

The experimental results provide clear evidence that environmental models that ignore broader-scope effects such as weather and orientation may not suffice to provide realistic plume propagation data. Detailed weather models, in conjunction with high fidelity atmospheric modeling, drive accurate simulation results for evaluating complex interactions between the environment and the sensor network.

Careful consideration of node placement in sensor network deployment is also required, as shown by simulation. Intelligent organization of sensor nodes providing adequate coverage while maintaining balanced node density in an area of interest can minimize side effects such as loss of information due to packet collisions. Moreover, these results emphasize the importance of utilizing a high fidelity sensor network simulator with accurate radio propagation models. Less realistic models can compromise the overall accuracy of the simulation by including unrealistic packet transmissions and receptions which, in turn, could affect other packets through collisions or dropped packets due to bandwidth limitations.

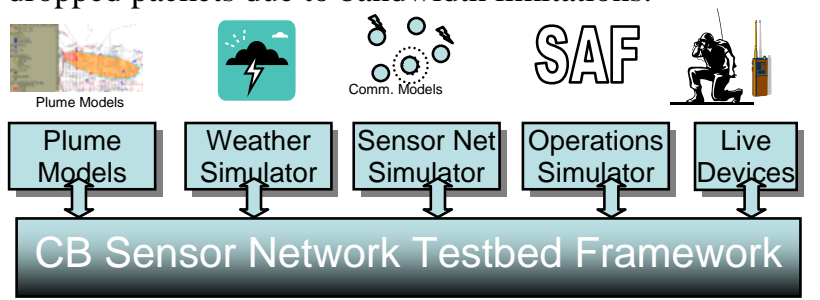

Figure 29: Generalized chemical/biological sensor network testbed framework

In general, although existing simulations of distinct but interdependent systems exist, it is our contention that a scenario-informed approach to simulation at highfidelity will expose unexpected behaviors that simpler assumptions will not capture. Our high-fidelity simulation-based evaluation approach can be generalized into an integrated environment of a rich variety of models, simulations and live equipment in a framework to configure, simulate/emulate, test and evaluate sensor-based designs, as shown in Figure 29. A unified, flexible Testing and Evaluation (T\&E) 
framework for chemical/biological (CB) sensors is envisioned which can be built on top of existing standards to the extent possible. Within this T\&E framework, the Department of Defense High Level Architecture (HLA) can be leveraged as a component for runtime integration to facilitate incorporation of existing simulators.

The interplay of CB dispersion, weather effects and sensor communications can be evaluated together ultimately in the context of actual operations. To this end, it is important to integrate them with operations simulators such as semi-automated forces (SAF) simulators, facilitating end-to-end, mission-specific evaluations. A similar need also exists in T\&E of live devices with respect to their operational response and effectiveness in the larger context. Due to the multitude and complexity of the sub-components, it is challenging to evolve a unified view of the interactions/dependencies among the components in the large CB sensor T\&E scenarios. Interaction between simulated and live components is challenging due to virtual/real conversion issues, especially for chemical/biological interactions, which need to be resolved. Proper identification of relevant interaction units is important for maximum flexibility.

Design of such a comprehensive framework enables a wide range of T\&E possibilities. Scenarios of operation can be effectively simulated and measures of performance can be obtained for individual subsystems or for the entire operation as a whole. Alternatively, any of the components can be evaluated against the others. For example, the performance of devices of interest can be evaluated in the larger context of operations, stimulated by CB dispersion according to plume models and its effects on operations simulated at the entity level (e.g. infantry). The environment can help uncover possible realms of operation that are otherwise hard to capture with small, isolated testing. Moreover, the backplane-based testbed environment not only permits flexible T\&E scenarios, but also helps control the levels of fidelity and scalability required for a given testing scenario.

\section{Acknowledgements}

The authors would like to thank Ronald Lee for information on SCIPUFF operation and Auroop Ganguly for background on related work in plume modeling.

\section{REFERENCES}

[1] R. R. Brooks and S. S. Iyengar, Multisensor Fusion: Fundamentals and Applications with Software: Prentice Hall, 1997.

[2] C. Huang, T. Hsing, N. Cressie, A. R. Ganguly, V. A. Protopopescu, and N. S. Rao, "Plume Model Identification Based on Statistcal Analysis of Sensor Network Data."

[3] L. Dan, K. D. Wong, H. Yu Hen, and A. M. Sayeed, "Detection, classification, and tracking of targets," Signal Processing Magazine, IEEE, vol. 19, pp. 17-29, 2002.

[4] R. R. Brooks, C. Griffin, and D. S. Friedlander, "Self-Organized Distributed Sensor Network Entity Tracking," International Journal of High Performance Computing, vol. 16, 2002.

[5] D. McErlean and S. Narayanan, "Distributed Detection and Tracking in Sensor Networks," presented at 36th Asilomar Conference on Signals, Systems \& Computers, 2002.

[6] G. Nofsinger and G. Cybenko, "Distributed Chemical Plume Process Detection: Milcom 2005 1644," presented at Military Communications Conference, 2005.

[7] R. I. Sykes, D. S. Henn, S. F. Parker, and R. S. Gabruk, "SCIPUFF - A generalized hazard dispersion model," presented at Ninth Joint Conference on the Applications of Air Pollution Meteorology with A\&WMA, 1996.

[8] R. I. Sykes, S. F. Parker, D. S. Henn, C. P. Cerasoli, and L. P. Santos, "PC-SCIPUFF Version 1.2PD Technical Documentation," Titan Corporation, Titan Research \& Technology Division, ARAP Group, P.O. Box 2229, Princeton, NJ, 08543-2229 ARAP Report No. 718, 1998.

[9] J. Hill, R. Szewczyk, A. Woo, H. Seth, D. Culler, and K. Pister, "System Architecture Directions for Networked Sensors," in Proceedings of the Ninth International Conference on Architectural Support for Programming Languages and Operating Systems. Cambridge, Massachusetts, United States: ACM Press, 2000.

[10]P. Levis, N. Lee, M. Welsh, and D. Culler, "TOSSIM: Accurate and Scalable Simulation of Entire TinyOS Applications," in Proceedings of the 1st International Conference on Embedded Networked Sensor Systems. Los Angeles, California, USA: ACM Press, 2003.

[11]E. Ould-Ahmed-Vall, G. F. Riley, B. S. Heck, and D. Reddy, "Simulation of large-scale sensor networks using GTSNetS," in Proceedings of the 13th IEEE International Symposium on Modeling, Analysis, and Simulation of Computer and Telecommunication Systems. Atlanta, GA, 2005, pp. 211-218.

[12]A. Sobeih and J. C. Hou, "A Simulation Framework for Sensor Networks in J-Sim," University of Illionis at Urbana-Champaign UIUCDCS-R-2003-2386, 2003.

[13] G. Chen, J. Branch, M. J. Pflug, L. Zhu, and B. Szymanski, "SENSE: A Sensor Network Simulator," in Advances in Pervasive Computing and Networking, B. Szymanksi and B. Yener, Eds.: Springer, 2004, pp. 249-267.

[14] P. Sung, S. Andreas, and B. S. Mani, "SensorSim: a simulation framework for sensor networks," in Proceedings of the 3rd ACM international workshop on Modeling, analysis and simulation of 
wireless and mobile systems. Boston, Massachusetts, United States: ACM Press, 2000.

[15] L. F. Perrone and D. M. Nicol, "A scalable simulator for TinyOS applications," presented at Winter Simulation Conference, 2002.

[16]L. Girod, J. Elson, A. Cerpa, T. Stathopoulos, N. Ramanathan, and D. Estrin, "EmStar: a Software Environment for Developing and Deploying Wireless Sensor Networks," presented at USENIX General Track 2004, 2004.

[17] G. Lewis, S. Thanos, R. Nithya, E. Jeremy, E. Deborah, O. Eric, and S. Tom, "A system for simulation, emulation, and deployment of heterogeneous sensor networks," in Proceedings of the 2nd international conference on Embedded networked sensor systems. Baltimore, MD, USA: ACM Press, 2004.

[18] M. Demmer, P. Levis, A. Joki, E. Brewer, and D. Culler, "Tython: A Dynamic Simulation Environment for Sensor Networks," UC Berkeley UCB/CSD-05-1372, 2005.

[19]"TinyOS Multihop Routing. http://www.tinyos.net/tinyos-

1.x/doc/multihop/multihop_routing.html."

[20]D. B. Turner, "Workbook of Atmospheric Dispersion Estimates," U.S. Department of Health, Education and Welfare, National Air Pollution Control Administration, Cincinnati, Ohio PHS Publication No. 999-AP-26, 1970.

[21] "User's Guide for the AMS/EPA Regulatory Model - AERMOD," U.S. Environmental Protection Agency EPA-454/B-03-001, 2004.

[22]"ALOHA: Areal Locations of Hazardous Atmospheres User's Manual," U.S. Environmental Protection Agency, National Oceanic and Atmospheric Administration 2006.

[23] "User's Guide for the Industrial Source Complex (ISC3) Dispersion Models," U.S. Environmental Protection Agency EPA-454/B-95-003a, 1995.

[24] R. R. Draxler, "Hybrid Single-Particle Lagrangian Integrated Trajectories (HY-SPLIT): Model Description," National Oceanic and Atmospheric Administration ERL ARL-166, 1988.

[25] J. S. Scire, D. G. Strimaitis, and R. J. Yamartino, "A User's Guide for the CALPUFF Dispersion Model," Earth Tech Inc., Concord, MA 2000.

[26] T. Yamada, S. Bunker, and M. Moss, "Numerical Simulations of Atmospheric Transport and Diffusion over Coastal Complex Terrain," Journal of Applied Meteorology, vol. 31, pp. 565-578, 1991.

[27] C.-H. Moeng, "A large-eddy-simulation model for the study of planetary boundary-layer turbulence," Journal of the Atmospheric Sciences, vol. 41, pp. 2052-2062, 1984.

[28]S. T. Chan, "FEM3C-An improved threedimensional heavy-gas dispersion model: User's manual," LLNL, Livermore, CA UCRL-MA116567 Rev. 1, 1994.
[29] F. Camelli and R. Löhner, "VLES Study of Flow and Dispersion Patterns in Heterogeneous Urban Areas," AIAA-06-1419, 2006. 